\title{
LEAVITT PATH ALGEBRAS AND DIRECT LIMITS
}

\author{
K. R. GOOdEARL
}

\begin{abstract}
An introduction to Leavitt path algebras of arbitrary directed graphs is presented, and direct limit techniques are developed, with which many results that had previously been proved for countable graphs can be extended to uncountable ones. Such results include characterizations of simplicity, characterizations of the exchange property, and cancellation conditions for the K-theoretic monoid of equivalence classes of idempotent matrices.
\end{abstract}

\section{INTRODUCTION}

The algebras of the title descend from algebras constructed by W. G. Leavitt [21] to exhibit rings in which free modules of specific different finite ranks are isomorphic. Later, and independently, J. Cuntz introduced an analogous class of $\mathrm{C}^{*}$-algebras [15]. Generalizations of these led to a large class of $\mathrm{C}^{*}$-algebras built from directed graphs, and the construction was carried to the algebraic category by G. Abrams and G. Aranda Pino [1]. For both historical and technical reasons, the graphs used in constructing graph $\mathrm{C}^{*}$-algebras and Leavitt path algebras have been assumed to be countable, although the construction does not require this. Our motivation for this paper was to initiate the study of Leavitt path algebras of uncountable graphs and to show that many (perhaps all) of the known theorems hold for uncountable as well as countable graphs.

The first section of the paper is an expository account of the basic ideas and constructions involved with Leavitt path algebras $L_{K}(E)$, where $K$ is a field and $E$ a (directed) graph. Further expository material is incorporated into the second section, where appropriate categories are defined in which direct limits exist. In particular, we show that $L_{K}(E)$ is a direct limit of Leavitt path algebras of certain countable subgraphs of $E$, over a countably directed set. This result allows "finitely definable" properties to be transferred from the countable to the uncountable case. Section 3 illustrates this procedure with various results concerning ideals. For instance, we extend a theorem of Tomforde [24] saying that the ideals of $L_{K}(E)$ are homogeneous (with respect to the canonical grading) if and only if $E$ satisfies the graph-theoretical condition (K), and a theorem of Abrams-Aranda [3] and Tomforde [op. cit.] characterizing simplicity of $L_{K}(E)$ in terms of graph-theoretical conditions on $E$. In Section 4, we extend another theorem of Abrams-Aranda [op. cit.], showing that $L_{K}(E)$ is an exchange ring if and only if $E$ satisfies condition (K). The

2000 Mathematics Subject Classification. 16S99, 16G20. 
final section addresses the abelian monoid $V(R)$ associated with any ring $R$, which can be built from either equivalence classes of idempotent matrices or isomorphism classes of finitely generated projective modules. We give an expository account of $V(R)$ in the general (nonunital) case, and develop some nonunital module category machinery in order to show that Morita equivalent idempotent rings have isomorphic $V$ s. This is needed in our final result, extending a theorem of Ara-Moreno-Pardo [10], which says that $V\left(L_{K}(E)\right)$ is always an unperforated, separative refinement monoid.

In order to keep this paper to a reasonable size, we have chosen to present only a sample of results where a countability hypothesis on the graph can be removed, and we have not addressed potential $\mathrm{C}^{*}$-algebra analogs of the methods. We invite readers to explore removing countability assumptions from other results. In particular, some natural candidates can be found in $[\mathbf{2}, \mathbf{3}, \mathbf{1 0}, \mathbf{1 1}, \mathbf{1 3}]$.

\section{BASICS}

In this section, we give some basic notation for graphs, path algebras, and Leavitt path algebras, and discuss a few basic results about these objects. For more on the historical background, we recommend Abrams' article in [14].

1.1. Graphs. A directed graph is a 4-tuple $E=\left(E^{0}, E^{1}, r_{E}, s_{E}\right)$ consisting of two disjoint sets $E^{0}, E^{1}$ and two maps $r_{E}, s_{E}: E^{1} \rightarrow E^{0}$. The adjective "directed" is often omitted, as are the subscripts on the maps $r_{E}$ and $s_{E}$, unless several graphs are under discussion at once. The elements of $E^{0}$ and $E^{1}$ are called the vertices and edges of $E$, respectively. If $e \in E^{1}$, then $s(e)$ and $r(e)$ are called the source and range of $e$, respectively. One also says that $e$ goes from $s(e)$ to $r(e)$, written $e: s(e) \rightarrow r(e)$ or drawn $s(e) \stackrel{e}{\rightarrow} r(e)$ in diagrams.

A directed path in $E$, usually just called a path, is a sequence of edges with the source of each edge matching the range of an adjacent edge. There are two conventions for orienting such sequences: right to left or left to right, and unfortunately both are in common use. Since the left to right convention is used in a majority of papers on Leavitt path algebras and graph $\mathrm{C}^{*}$-algebras, we follow that convention here. Thus, a path in $E$ consists of a sequence $e_{1}, e_{2}, \ldots, e_{n}$ of edges from $E^{1}$ such that $r\left(e_{i}\right)=s\left(e_{i+1}\right)$ for all $i=1, \ldots, n-1$. We shall write such a path as a product $p=e_{1} e_{2} \cdots e_{n}$, labelling $s(p)=s\left(e_{1}\right)$ and $r(p)=r\left(e_{n}\right)$. The length of a path is the number of edges it contains. It is important to allow a path of length zero at each vertex $v$; this is a path with source and range $v$, including no edges, and the natural notation for this path is just $v$.

1.2. Duals and Doubles. The dual of a graph $E$ is a graph $E^{*}$ consisting of the same vertices as $E$ but with all edges reversed. Specifically,

- $\left(E^{*}\right)^{0}=E^{0}$;

- $\left(E^{*}\right)^{1}$ is a set $\left(E^{1}\right)^{*}=\left\{e^{*} \mid e \in E^{1}\right\}$ which is in bijection with $E^{1}$ via $e \mapsto e^{*}$;

- $r\left(e^{*}\right)=s(e)$ and $s\left(e^{*}\right)=r(e)$ for all $e \in E^{1}$.

The $*$ notation is extended from edges to paths in the obvious manner. Thus, if $p=$ $e_{1} e_{2} \cdots e_{n}$ is a path in $E$ from a vertex $v$ to a vertex $w$, then $p^{*}=e_{n}^{*} e_{n-1}^{*} \cdots e_{1}^{*}$ is a path in $E^{*}$ from $w$ to $v$. (Note the length zero case: $v^{*}=v$ for $v \in E^{0}$.) 
It is assumed (sometimes only tacitly) that the set $\left(E^{1}\right)^{*}$ is disjoint from $E^{0} \sqcup E^{1}$. Then the double (or extended graph) of $E$, denoted $\widehat{E}$ or $D(E)$, is the union of $E$ and $E^{*}$. Thus, $\widehat{E}^{0}=E^{0}$ and $\widehat{E}^{1}=E^{1} \sqcup\left(E^{1}\right)^{*}$, with range and source maps $r_{\widehat{E}}$ and $s_{\widehat{E}}$ combining those of $E$ and $E^{*}$. When working with $\widehat{E}$, the edges (paths) from $E^{1}$ are often called real edges (real paths) and those from $\left(E^{1}\right)^{*}$ ghost edges (ghost paths).

1.3. Path Algebras. Let $E$ be a graph and $K$ a field. The path algebra of $E$ over $K$, denoted $K E$, is the $K$-algebra based on the vector space over $K$ with basis the set of all paths in $E$, and with multiplication induced from concatenation of paths: if $p=e_{1} e_{2} \cdots e_{n}$ and $q=f_{1} f_{2} \cdots f_{m}$ are paths in $E$, their product in $K E$ is given by

$$
p q= \begin{cases}e_{1} e_{2} \cdots e_{n} f_{1} f_{2} \cdots f_{m} & \left(\text { if } r\left(e_{n}\right)=s\left(f_{1}\right)\right) \\ 0 & \text { (otherwise) }\end{cases}
$$

We shall need the observation that $K E$ is the $K$-algebra presented by generators from the set $E^{0} \sqcup E^{1}$ with the following relations:

- $v^{2}=v$ for all $v \in E^{0}$;

- $v w=0$ for all distinct $v, w \in E^{0}$;

- $e=s(e) e=e r(e)$ for all $e \in E^{1}$.

The path algebra $K E$ is unital if and only if $E^{0}$ is finite, in which case the identity is the sum of the vertices (= paths of length zero) in $E$. In general, $K E$ is a ring with local units, meaning that $K E$ contains a set $Q$ of pairwise commuting idempotents such that for each $x \in K E$, there is some $q \in Q$ with $q x=x q=x$. Namely, take $Q$ to be the set of all finite sums of distinct vertices of $E$.

1.4. Leavitt Path Algebras. Before defining these algebras, we recall two standard graph-theoretic concepts. A vertex $v$ in a graph $E$ is a sink if $v$ emits no edges, i.e., there are no edges $e \in E^{1}$ with $s(e)=v$. At the other extreme, $v$ is an infinite emitter if there are infinitely many edges $e \in E^{1}$ with $s(e)=v$.

The Leavitt path algebra of $E$ over a field $K$, denoted $L_{K}(E)$, is the quotient of the path algebra $K \widehat{E}$ modulo the ideal generated by the following elements:

- $e^{*} e-r(e)$, for all $e \in E^{1}$;

- $e^{*} f$, for all distinct $e, f \in E^{1}$;

- $v-\sum_{e \in E^{1}, s(e)=v} e e^{*}$, for every $v \in E^{0}$ which is neither a sink nor an infinite emitter.

The corresponding equations in $L_{K}(E)$ are known as the Cuntz-Krieger relations. Note that $L_{K}(E)$ is a ring with local units.

It is standard practice to use the same names for vertices, edges, and paths in $\widehat{E}$ as for their cosets in $L_{K}(E)$. This introduces no ambiguities when working with real paths or ghost paths (see Lemmas 1.5 and 1.6 below), but care must be taken with paths involving both real and ghost edges. For instance, if $e \in E^{1}$, then $e^{*} e$ denotes a path of length 2 in $K \widehat{E}$, while $e^{*} e=r(e)$ in $L_{K}(E)$. Let us reserve the symbol $\pi_{K, E}$ for the quotient map $K \widehat{E} \rightarrow L_{K}(E)$, for use when it is important to distinguish cosets from their representatives. 
Since the Cuntz-Krieger relations can be used to reduce any expression involving a product of a ghost path followed by a real path, all elements of $L_{K}(E)$ can be written as $K$-linear combinations of products $p q^{*}$ where $p$ and $q$ are real paths in $E$.

The path algebra $K \widehat{E}$ supports a $\mathbb{Z}$-grading under which real edges have degree 1 while ghost edges have degree -1 . Thus, a real path $p$ is homogeneous of degree length $(p)$, while a ghost path $p^{*}$ is homogeneous of degree $-\operatorname{length}(p)$. The relations used to form $L_{K}(E)$ from $K \widehat{E}$ are all homogeneous (of degree zero), and therefore $L_{K}(E)$ inherits an induced $\mathbb{Z}$-grading.

It has been noted in many papers that the vertices of a graph are linearly independent when viewed as elements in a Leavitt path algebra, but this fact does not appear to have been explicitly proved in the literature. We take the opportunity to do so here.

Lemma 1.5. Let $K$ be a field and $E$ a graph. The quotient map $\pi=\pi_{K, E}: K \widehat{E} \rightarrow L_{K}(E)$ sends the vertices from $E^{0}$ to $K$-linearly independent elements of $L_{K}(E)$.

Proof. We proceed by building a representation of $L_{K}(E)$ as linear transformations on a vector space.

Let $\aleph$ be an infinite cardinal at least as large as $\operatorname{card}\left(E^{0} \sqcup E^{1}\right)$. Let $X$ be an $\aleph$ dimensional vector space over $K$, and set $R=\operatorname{End}_{K}(X)$. Since $\aleph \cdot \operatorname{card}\left(E^{0}\right)=\aleph$, we can choose a decomposition $X=\bigoplus_{v \in E^{0}} X_{v}$ with $\operatorname{dim} X_{v}=\aleph$ for all $v$. For $v \in E^{0}$, let $p_{v} \in R$ denote the projection of $X$ onto $X_{v}$ with kernel $\bigoplus_{w \neq v} X_{w}$. For each $v \in E^{0}$ which is not a sink, we can choose a decomposition $X_{v}=\bigoplus_{e \in E^{1}, s(e)=v} Y_{e}$ with $\operatorname{dim} Y_{e}=\aleph$ for all $e$. For $e \in E^{1}$, let $q_{e} \in R$ denote the projection of $X$ onto $Y_{e}$ with kernel

$$
\left(1-p_{s(e)}\right) X \oplus \bigoplus_{\substack{f \in E^{1}, f \neq e \\ s(f)=s(e)}} Y_{f}
$$

Finally, for each $e \in E^{1}$, choose $\alpha_{e} \in q_{e} R p_{r(e)}$ and $\alpha_{e}^{*} \in p_{r(e)} R q_{e}$ such that $\alpha_{e}$ restricts to an isomorphism $X_{r(e)} \rightarrow Y_{e}$ and $\alpha_{e}^{*}$ restricts to the inverse isomorphism.

Observe that $p_{v}^{2}=p_{v}$ and $p_{v} p_{w}=0$ for all distinct $v, w \in E^{0}$. For all $e \in E^{1}$, we have $\alpha_{e}=p_{s(e)} \alpha_{e}=\alpha_{e} p_{r(e)}$ and $\alpha_{e}^{*}=p_{s\left(e^{*}\right)} \alpha_{e}^{*}=\alpha_{e}^{*} p_{r\left(e^{*}\right)}$. Consequently, there is a unique $K$-algebra homomorphism $\phi: K \widehat{E} \rightarrow R$ such that

- $\phi(v)=p_{v}$ for all $v \in E^{0}$;

- $\phi(e)=\alpha_{e}$ and $\phi\left(e^{*}\right)=\alpha_{e}^{*}$ for all $e \in E^{1}$.

We next check that ker $\phi$ contains the defining relations of $L_{K}(E)$. First, given $e \in E^{1}$, observe that $\alpha_{e}^{*} \alpha_{e}=p_{r(e)}$, whence $e^{*} e-r(e) \in \operatorname{ker} \phi$. If $e, f \in E^{1}$ are distinct, then $\alpha_{e}^{*} \alpha_{f}=\alpha_{e}^{*} q_{e} q_{f} \alpha_{f}=0$, whence $e^{*} f \in \operatorname{ker} \phi$. Finally, if $v \in E^{0}$ is neither a sink nor an infinite emitter, then

$$
p_{v}=\sum_{e \in E^{1}, s(e)=v} q_{e}=\sum_{e \in E^{1}, s(e)=r} \alpha_{e} \alpha_{e}^{*},
$$

whence $v-\sum_{e \in E^{1}, s(e)=r} e e^{*} \in \operatorname{ker} \phi$. Thus, $\phi$ induces a unique $K$-algebra homomorphism $\psi: L_{K}(E) \rightarrow R$ such that $\psi \pi=\phi$. 
By construction, the projections $p_{v}$ for $v \in E^{0}$ are pairwise orthogonal nonzero idempotents, and hence $K$-linearly independent elements of $R$. Since $\psi \pi(v)=p_{v}$ for all $v$, we conclude that $\pi$ indeed maps the elements of $E^{0}$ to $K$-linearly independent elements of $L_{K}(E)$.

That the result of Lemma 1.5 extends to (real) paths is proved in [23, Lemma 1.1]. It is easy to include ghost paths, as follows.

Lemma 1.6. Let $K$ be a field and $E$ a graph. The quotient map $\pi=\pi_{K, E}: K \widehat{E} \rightarrow L_{K}(E)$ restricts to an embedding of the subspace $K E+K E^{*}$ of $K \widehat{E}$ into $L_{K}(E)$.

Proof. For purposes of this proof, let us write $\bar{x}=\pi(x)$ for $x \in K \widehat{E}$. We need to show that if

$$
\sum_{i=1}^{m} \alpha_{i} \overline{p_{i}}+\sum_{j=1}^{n} \beta_{j} \overline{q_{j}^{*}}=0
$$

for distinct real paths $p_{i}$, distinct ghost paths $q_{j}^{*}$ of positive length, and scalars $\alpha_{i}, \beta_{j} \in K$, then $\alpha_{i}=\beta_{j}=0$ for all $i, j$. If $n=0$ and all the $p_{i}$ have length zero, this follows from Lemma 1.5.

Since $L_{K}(E)$ is a $\mathbb{Z}$-graded algebra, the equation $(\dagger)$ breaks into homogeneous components, which can be treated separately. Hence, we may assume that either $m=0$ or $n=0$, that all the $p_{i}$ have the same length, and that all the $q_{j}$ have the same length. Further, $(\dagger)$ breaks into separate equations of similar form when the terms are multiplied on the left by $\bar{v}$ and on the right by $\bar{w}$, for any vertices $v, w \in E^{0}$. Consequently, it suffices to consider the case where all the $p_{i}$ and $q_{j}^{*}$ have the same source $v$ and the same range $w$.

Next, assume that $n=0$. The short argument of [23, Lemma 1.1] applies here; we repeat it for the reader's convenience. Since the $p_{i}$ are distinct paths of the same length, from $v$ to $w$, we have $\overline{p_{j}^{*}} \overline{p_{i}}=\delta_{i j} \bar{w}$ for all $i, j$. Hence, $\alpha_{i} \bar{w}=0$ for all $i$. However, $\bar{w} \neq 0$ by Lemma 1.5, and therefore all $\alpha_{i}=0$.

The case $m=0$ is handled in the same manner. Namely, since the $q_{j}$ are distinct paths of the same length, from $w$ to $v$, we have $\bar{q}_{j}^{*} \bar{q}_{i}=\delta_{i j} \bar{v}$ for all $i, j$. It follows that all $\beta_{j}=0$, completing the proof.

1.7. Opposites. The opposite algebras of path algebras and Leavitt path algebras are easily understood in terms of dual graphs. If $K$ is a field, $E$ a graph, and the notation $*$ is extended from edges to paths as in (1.2), then $*$ provides a bijection from the standard basis of $K E$ (the set of paths in $E$ ) onto the standard basis of $K E^{*}$ (the set of paths in $\left.E^{*}\right)$. Consequently, $*$ extends uniquely to a $K$-vector space isomorphism of $K E$ onto $K E^{*}$. Since $*$ reverses the composition of edges in paths, it also reverses multiplication of paths. Therefore $*: K E \rightarrow K E^{*}$ is a $K$-algebra anti-isomorphism. In particular, it follows that $K E^{*}$ is isomorphic to the opposite algebra of $K E$.

The graph $\widehat{E}$ is self-dual, in that $\widehat{E} \cong \widehat{E}^{*}$, by an isomorphism that fixes vertices and sends $e \mapsto\left(e^{*}\right)^{*}$ and $e^{*} \mapsto e^{*}$ for $e \in E^{1}$. It is convenient to treat this isomorphism as an identification, by setting $\left(e^{*}\right)^{*}=e$ for all real edges $e$. Extending this to paths in the 
natural manner, we obtain a $K$-algebra anti-automorphism of $K \widehat{E}$, still denoted $*$. In particular, $K \widehat{E}$ is isomorphic to its opposite algebra.

Since the set of generators for the kernel of the quotient map $\pi_{K, E}$ given in (1.4) is mapped onto itself by $*$, this ideal is invariant under $*$. Therefore $*$ induces a $K$-algebra anti-automorphism of $L_{K}(E)$, which we also denote $*$. Hence, $L_{K}(E)$ is isomorphic to its opposite.

1.8. Reversing the Path Composition Convention. At the level of path algebras, reversing the convention for writing and composing paths just changes the algebra to its opposite, but this is not so for Leavitt path algebras. Just for the present subsection, let us write $K \downarrow E$ to denote the path algebra of $E$ over $K$ constructed using the right to left convention for paths. Thus, a path in $K \uparrow E$ is a product $p=e_{n} e_{n-1} \cdots e_{1}$ where $e_{1}, \ldots, e_{n}$ are edges from $E^{1}$ such that $r\left(e_{i}\right)=s\left(e_{i+1}\right)$ for $i=1, \ldots, n-1$. Such a path runs from $s(p)=s\left(e_{1}\right)$ to $r(p)=r\left(e_{n}\right)$. Multiplication of paths $p$ and $q$ in $K \uparrow E$ follows the rule that $p q=0$ unless $r(q)=s(p)$, in which case $p q$ is the concatenation of $p$ and $q$ in the order "first $q$, then $p$ ". Observe that $K \uparrow E$ is the $K$-algebra presented by generators from the set $E^{0} \sqcup E^{1}$ with the following relations:

- $v^{2}=v$ for all $v \in E^{0}$;

- $v w=0$ for all distinct $v, w \in E^{0}$;

- $e=r(e) e=e s(e)$ for all $e \in E^{1}$.

It follows that $K \uparrow E$ equals the opposite algebra of $K E$, and so $K \uparrow E \cong K E^{*}$.

The construction of Leavitt path algebras requires choices of two conventions - one for paths, and one for the Cuntz-Krieger relations. If we change both conventions from the ones in (1.1) and (1.4), we obtain the quotient of $K \downarrow E$ modulo the ideal generated by

- $e e^{*}-r(e)$, for all $e \in E^{1}$;

- $f e^{*}$, for all distinct $e, f \in E^{1}$;

- $v-\sum_{e \in E^{1}, s(e)=v} e^{*} e$, for every $v \in E^{0}$ which is neither a sink nor an infinite emitter.

This algebra is just the opposite algebra of $L_{K}(E)$, and so it is isomorphic to $L_{K}(E)$.

However, if we adopt the right to left convention for paths without reversing products in the Cuntz-Krieger relations, the latter, to be sensible, must be modified by interchanging sources and ranges. This time, we obtain the quotient of $K \downarrow E$ modulo the ideal generated by

- $e^{*} e-s(e)$, for all $e \in E^{1}$;

- $e^{*} f$, for all distinct $e, f \in E^{1}$;

- $v-\sum_{e \in E^{1}, r(e)=v} e e^{*}$, for every $v \in E^{0}$ which is neither a source nor an infinite receiver.

Since $s(e)=r\left(e^{*}\right)$ and $r(e)=s\left(e^{*}\right)$ for $e \in E^{1}$, the opposite of the above algebra is naturally isomorphic to $L_{K}\left(E^{*}\right)$, and hence the algebra itself is isomorphic to $L_{K}\left(E^{*}\right)$. Thus, to transfer results proved about Leavitt path algebras constructed with these conventions, one must dualize any graph conditions that appear.

Much of the initial work on Leavitt path algebras restricted attention to graphs of the 
following type.

1.9. Row-Finite Graphs. The incidence matrix for a graph $E$ is an $E^{0} \times E^{0}$ matrix in which the $(v, w)$-entry (for vertices $v, w \in E^{0}$ ) is the number of edges from $v$ to $w$ in $E^{1}$. We say that $E$ is a row-finite graph provided its incidence matrix is row-finite with finite entries, i.e., each row contains at most finitely many nonzero entries, none of which is $\infty$. Thus, $E$ is row-finite if and only if each vertex of $E$ emits at most finitely many edges.

\section{DiRECT Limits}

Here we set up an appropriate category of graphs on which the construction of Leavitt path algebras (over a given field) is functorial, and show that this functor preserves direct limits. It is helpful to base the discussion on direct limits of ordinary path algebras, and so we begin with functoriality of the path algebra construction. Recall that the term direct limit (equivalently, inductive limit) refers to a colimit (in a category) over a system of objects and morphisms indexed by a directed set.

2.1. The Directed Graph Category. A graph morphism from a graph $E$ to a graph $F$ is a pair $\phi=\left(\phi^{0}, \phi^{1}\right)$ consisting of maps $\phi^{0}: E^{0} \rightarrow F^{0}$ and $\phi^{1}: E^{1} \rightarrow F^{1}$ such that $s_{F} \phi^{1}=\phi^{0} s_{E}$ and $r_{F} \phi^{1}=\phi^{0} r_{E}$. Due to the assumption that $E^{0} \cap E^{1}=F^{0} \cap F^{1}=\varnothing$, we can view $\phi$ as a function $E^{0} \sqcup E^{1} \rightarrow F^{0} \sqcup F^{1}$ that restricts to $\phi^{0}$ and $\phi^{1}$.

Particularly useful graph morphisms arise when $E$ is a subgraph of $F$, meaning that $E$ consists of some of the vertices and edges of $F$. Of course, a collection of vertices and edges from $F$ does not naturally form a graph unless the source and range vertices of the chosen edges are included among the chosen vertices. Thus, to say that $E$ is a subgraph of $F$ means that

- $E^{0} \subseteq F^{0}$ and $E^{1} \subseteq F^{1}$

- $r_{F}(e), s_{F}(e) \in E^{0}$ for all $e \in E^{1}$;

- $r_{E}=\left.r_{F}\right|_{E^{1}}$ and $s_{E}=\left.s_{F}\right|_{E^{1}}$.

When $E$ is a subgraph of $F$, the inclusion map $E^{0} \sqcup E^{1} \rightarrow F^{0} \sqcup F^{1}$ forms a graph morphism $E \rightarrow F$.

We shall let DiGr denote the category of directed graphs: the objects of DiGr are arbitrary directed graphs, and the morphisms are arbitrary graph morphisms.

The construction of path algebras over a field $K$ appears functorial at first glance, but there is one problem with preservation of relations. For example, consider the graph morphism

$$
\phi: E=(v \stackrel{e}{\longrightarrow} w) \longrightarrow F=(x \stackrel{f}{5})
$$

sending the vertices $v, w \in E^{0}$ to the vertex $x \in F^{0}$, and the edge $e \in E^{1}$ to the edge $f \in F^{1}$. Since $v w=0$ in $K E$ while $x^{2}=x \neq 0$ in $K F$, there is no $K$-algebra morphism $K E \rightarrow K F$ extending $\phi$. To avoid this problem, we restrict attention to graph morphisms which are injective on vertices. 
2.2. Path Algebra Functors. Let $\mathbf{D i G r}_{0}$ be the subcategory of $\mathbf{D i G r}$ whose objects are arbitrary directed graphs and whose morphisms are those graph morphisms $\phi$ for which $\phi^{0}$ is injective. Given a field $K$, let $K$-Alg denote the category of (not necessarily unital) $K$ algebras. We allow completely arbitrary $K$-algebras as objects of $K$-Alg (that is, arbitrary vector spaces over $K$, equipped with associative, $K$-bilinear multiplications), and arbitrary $K$-algebra morphisms between them (that is, arbitrary multiplicative $K$-linear maps). Any direct system in $K$-Alg has a direct limit in this category.

Now if $\phi$ is a morphism in $\mathbf{D i G r} \mathbf{r}_{0}$, we have

- $\phi^{0}(v)^{2}=\phi^{0}(v)$ in $K F$ for all $v \in E^{0}$ (because $\phi^{0}$ sends vertices to vertices);

- $\phi^{0}(v) \phi^{0}(w)=0$ in $K F$ for all distinct $v, w \in E^{0}$ (because $\phi^{0}$ sends distinct vertices to distinct vertices);

- $\phi^{1}(e)=\phi^{0} s(e) \phi^{1}(e)=\phi^{1}(e) \phi^{0} r(e)$ in $K F$ for all $e \in E^{1}$ (because $\phi^{0} s(e)=s\left(\phi^{1}(e)\right)$ and $\left.\phi^{0} r(e)=r\left(\phi^{1}(e)\right)\right)$.

Consequently, the map $\phi: E^{0} \sqcup E^{1} \rightarrow F^{0} \sqcup F^{1}$ uniquely extends to a $K$-algebra morphism $K \phi: K E \rightarrow K F$.

The assignments $E \mapsto K E$ and $\phi \mapsto K \phi$ define a functor $K[-]: \mathbf{D i G r}_{0} \rightarrow K$-Alg.

There are additional problems with the construction of Leavitt path algebras over a field $K$, because the induced morphisms between path algebras of doubles do not always preserve Cuntz-Krieger relations. For instance, consider the graph morphism

$$
\psi: E=(v \stackrel{e}{\longrightarrow} w) \longrightarrow F=\left(v^{\prime} \stackrel{f}{\longrightarrow} w^{\prime}\right)
$$

sending $v \mapsto v^{\prime}$ and $w \mapsto w^{\prime}$, while $e \mapsto f$. Now $e e^{*}=v$ in $L_{K}(E)$, while $f f^{*}=v^{\prime}-g g^{*} \neq v^{\prime}$ in $L_{K}(F)$ (e.g., $g^{*}\left(g g^{*}\right) g=w^{\prime} \neq 0$, so $g g^{*} \neq 0$ ). Thus, there is no $K$-algebra morphism $L_{K}(E) \rightarrow L_{K}(F)$ extending $\psi$. Similarly, the graph morphism

$$
F=\left(v^{\prime} \stackrel{f}{\longrightarrow} w^{\prime}\right) \longrightarrow E=(v \stackrel{e}{\longrightarrow} w)
$$

sending $v^{\prime} \mapsto v$ and $w^{\prime} \mapsto w$, while $f, g \mapsto e$, does not extend to a $K$-algebra morphism $L_{K}(F) \rightarrow L_{K}(E)$, because $f^{*} g=0$ in $L_{K}(F)$ while $e^{*} e=w \neq 0$ in $L_{K}(E)$.

These problems can be dealt with by further restricting the allowable morphisms between graphs.

2.3. The Cuntz-Krieger Graph Category. Let us say that a graph morphism $\phi$ : $E \rightarrow F$ is a CK-morphism (short for Cuntz-Krieger morphism) provided

(1) $\phi^{0}$ and $\phi^{1}$ are both injective;

(2) For each $v \in E^{0}$ which is neither a sink nor an infinite emitter, $\phi^{1}$ induces a bijection $s_{E}^{-1}(v) \rightarrow s_{F}^{-1}\left(\phi^{0}(v)\right)$. 
In particular, condition (1) says that $\phi$ maps $E$ isomorphically onto a subgraph of $F$, while condition (2) implies that $\phi^{0}$ must send non-sink finite emitters to non-sink finite emitters.

If $E$ is row-finite, injectivity of $\phi^{0}$ together with condition (2) is sufficient to ensure injectivity of $\phi^{1}$. Thus, in this case, $\phi$ is a CK-morphism if and only if it is a complete graph homomorphism in the sense of [10, p. 161].

We shall say that a subgraph $E$ of a graph $F$ is a $C K$-subgraph provided the inclusion map $E \rightarrow F$ is a CK-morphism.

Now define $\mathbf{C K G r}$ to be the subcategory of $\mathbf{D i G r}$ whose objects are arbitrary directed graphs and whose morphisms are arbitrary CK-morphisms.

2.4. Leavitt Path Algebra Functors. Fix a field $K$, and consider a CK-morphism $\phi: E \rightarrow F$ between graphs $E$ and $F$. Now $\phi$ extends to a graph morphism $\widehat{\phi}: \widehat{E} \rightarrow \widehat{F}$ sending $e^{*} \mapsto \phi^{1}(e)^{*}$ for all $e \in E^{1}$, and $\widehat{\phi}^{0}=\phi^{0}$ is injective by assumption. Hence, $\widehat{\phi}$ uniquely induces a $K$-algebra morphism $K \widehat{\phi}: K \widehat{E} \rightarrow K \widehat{F}$. Observe that

- $\widehat{\phi}^{1}\left(e^{*}\right) \widehat{\phi}^{1}(e)=r\left(\widehat{\phi}^{1}(e)\right)=\widehat{\phi}^{0}(r(e))$ in $L_{K}(F)$ for all $e \in E^{1}$;

- $\widehat{\phi}^{1}\left(e^{*}\right) \widehat{\phi}^{1}(f)=0$ in $L_{K}(F)$ for all distinct $e, f \in E^{1}$ (because $\widehat{\phi}^{1}(e)$ and $\widehat{\phi}^{1}(f)$ are distinct edges in $F$ );

- For every $v \in E^{0}$ which is neither a sink nor an infinite emitter, the same properties hold for $\widehat{\phi}^{0}(v)$, and $\widehat{\phi}^{0}(v)=\sum_{f \in F^{1}, s(f)=\widehat{\phi}^{0}(v)} f f^{*}=\sum_{e \in E^{1}, s(e)=v} \widehat{\phi}^{1}(e) \widehat{\phi}^{1}\left(e^{*}\right)$ in $L_{K}(F)$ (because of condition $\left.(2.3)(2)\right)$.

Consequently, $K \widehat{\phi}$ uniquely induces a $K$-algebra morphism $L_{K}(\phi): L_{K}(E) \rightarrow L_{K}(F)$.

The assignments $E \mapsto L_{K}(E)$ and $\phi \mapsto L_{K}(\phi)$ define a functor $L_{K}: \mathbf{C K G r} \rightarrow K$-Alg.

Lemma 2.5. (a) Arbitrary direct limits exist in the categories $\mathbf{D i G r}, \mathbf{D i G r} \mathbf{r}_{0}$, and $\mathbf{C K G r}$.

(b) For any field $K$, the functors $K[-]: \mathbf{D i G r} \mathbf{r}_{0} \rightarrow K$-Alg and $L_{K}: \mathbf{C K G r} \rightarrow K-\mathbf{A l g}$ preserve direct limits.

Proof. (a) Let $\mathcal{E}=\left(\left(E_{i}\right)_{i \in I},\left(\phi_{i j}\right)_{i \leq j \text { in } I}\right)$ be a direct system in DiGr. Let $E_{\infty}^{0}$ and $E_{\infty}^{1}$ denote the direct limits of the corresponding direct systems of sets, $\left(\left(E_{i}^{0}\right)_{i \in I},\left(\phi_{i j}^{0}\right)_{i \leq j \text { in } I}\right)$ and $\left(\left(E_{i}^{1}\right)_{i \in I},\left(\phi_{i j}^{1}\right)_{i \leq j \text { in } I}\right)$, with limit maps $\eta_{i}^{0}: E_{i}^{0} \rightarrow E_{\infty}^{0}$ and $\eta_{i}^{1}: E_{i}^{1} \rightarrow E_{\infty}^{1}$.

Given $f \in E_{\infty}^{1}$, the set $I_{f}=\left\{i \in I \mid f \in \eta_{i}^{1}\left(E_{i}^{1}\right)\right\}$ is nonempty and upward directed. For any $i, j \in I_{f}$ and any $e \in\left(\eta_{i}^{1}\right)^{-1}(\{f\})$ and $e^{\prime} \in\left(\eta_{j}^{1}\right)^{-1}(\{f\})$, we have $\eta_{i}^{1}(e)=f=\eta_{j}^{1}\left(e^{\prime}\right)$, and so there is an index $k \geq i, j$ in $I$ such that $\phi_{i k}^{1}(e)=\phi_{j k}^{1}\left(e^{\prime}\right)$. Consequently,

$$
\eta_{i}^{0}(r(e))=\eta_{k}^{0} \phi_{i k}^{0}(r(e))=\eta_{k}^{0}\left(r\left(\phi_{i k}^{1}(e)\right)\right)=\eta_{k}^{0}\left(r\left(\phi_{j k}^{1}\left(e^{\prime}\right)\right)\right)=\eta_{j}^{0}\left(r\left(e^{\prime}\right)\right) .
$$

Thus, there is a unique well-defined map $r_{\infty}: E_{\infty}^{1} \rightarrow E_{\infty}^{0}$ such that $r_{\infty}\left(\eta_{i}^{1}(e)\right)=\eta_{i}^{0}(r(e))$ for all $i \in I$ and $e \in E_{i}^{1}$. Similarly, there is a unique well-defined map $s_{\infty}: E_{\infty}^{1} \rightarrow E_{\infty}^{0}$ such that $s_{\infty}\left(\eta_{i}^{1}(e)\right)=\eta_{i}^{0}(s(e))$ for all $i \in I$ and $e \in E_{i}^{1}$.

Now $E_{\infty}=\left(E_{\infty}^{0}, E_{\infty}^{1}, r_{\infty}, s_{\infty}\right)$ is a directed graph, and the maps $\eta_{i}=\eta_{i}^{0} \sqcup \eta_{i}^{1}: E_{i} \rightarrow E_{\infty}$ are morphisms in DiGr such that $\eta_{j} \phi_{i j}=\eta_{i}$ for all $i \leq j$ in $I$. It is routine to check that $E_{\infty}$, together with the maps $\eta_{i}$, is a direct limit for the system $\mathcal{E}$ in $\mathbf{D i G r}$.

If all the maps $\phi_{i j}^{0}$ are injective, then so are the maps $\eta_{i}^{0}$, and $E_{\infty}$ is a direct limit for $\mathcal{E}$ in $\mathrm{DiGr}_{0}$. 
Now assume that all the $\phi_{i j}$ are CK-morphisms, and note that the maps $\eta_{i}^{0}$ and $\eta_{i}^{1}$ are injective. Consider a vertex $v \in E_{\infty}^{0}$ which is neither a sink nor an infinite emitter. Then $v=\eta_{i}^{0}(w)$ for some $i \in I$ and some $w \in E_{i}^{0}$. Since $\eta_{i}^{1}$ is injective, we see that $w$ cannot be an infinite emitter. There is at least one edge $e \in E_{\infty}^{1}$ emitted by $v$, and, after possibly increasing $i$, we may assume that $e=\eta_{i}^{1}(f)$ for some edge $f \in E_{i}^{1}$ emitted by $w$. Hence, $w$ is not a sink. For all $j \geq i$ in $I$, the map $\phi_{i j}^{1}$ sends the edges emitted by $w$ bijectively onto the edges emitted by $\phi_{i j}^{0}(w)$, and consequently $\eta_{i}^{1}$ sends the edges emitted by $w$ bijectively onto the edges emitted by $v$. Thus, the $\eta_{i}$ are CK-morphisms.

Suppose we are given a graph $F$ and CK-morphisms $\theta_{i}: E_{i} \rightarrow F$ such that $\theta_{j} \phi_{i j}=\theta_{i}$ for all $i \leq j$ in $I$. Since $E_{\infty}$ is a direct limit for $\mathcal{E}$ in $\mathbf{D i G r} \mathbf{r}_{0}$, there is at least a unique graph morphism $\sigma: E_{\infty} \rightarrow F$ such that $\sigma \eta_{i}=\theta_{i}$ for all $i \in I$. In the same manner as above, one checks that $\sigma$ is a CK-morphism. Therefore $E_{\infty}$ is a direct limit for $\mathcal{E}$ in $\mathbf{C K G r}$.

(b) Let $\mathcal{E}=\left(\left(E_{i}\right)_{i \in I},\left(\phi_{i j}\right)_{i \leq j \text { in } I}\right)$ be a direct system in $\mathbf{D i G r}$, and construct the graph $E_{\infty}$ and maps $\eta_{i}$ as in part (a). We first show that $K E_{\infty}$ is a direct limit for the system $K[\mathcal{E}]=\left(\left(K E_{i}\right)_{i \in I},\left(K \phi_{i j}\right)_{i \leq j \text { in } I}\right)$ in $K$-Alg.

Let a $K$-algebra $A$, with limit maps $\theta_{i}: K E_{i} \rightarrow A$, be a direct limit for the system $K[\mathcal{E}]$. Since $\left(K \eta_{j}\right)\left(K \phi_{i j}\right)=K \eta_{i}$ for all $i \leq j$ in $I$, there is a unique $K$-algebra morphism $\psi: A \rightarrow K E_{\infty}$ such that $\psi \theta_{i}=K \eta_{i}$ for all $i \in I$. We show that $\psi$ is an isomorphism.

Any element $x \in K E_{\infty}$ is a $K$-linear combination of paths involving finitely many edges from $E_{\infty}^{1}$. The edges needed can all be found in $\eta_{i}^{1}\left(E_{i}^{1}\right)$ for some $i \in I$, whence $x \in\left(K \eta_{i}\right)\left(K E_{i}\right) \subseteq \psi(A)$. Consequently, $\psi$ is surjective. Now consider $a \in$ ker $\psi$, write $a=\theta_{i}(b)$ for some $i \in I$ and $b \in K E_{i}$, and write $b=\sum_{l \in L} \alpha_{l} p_{l}$ for some finite index set $L$, some $\alpha_{l} \in K$, and some paths $p_{l} \in E_{i}^{1}$. Then

$$
\sum_{l \in L} \alpha_{l}\left(K \eta_{i}\right)\left(p_{l}\right)=\psi \theta_{i}(b)=0
$$

where the $\left(K \eta_{i}\right)\left(p_{l}\right)$ can be viewed as paths in $E_{\infty}$. It follows that there is a partition $L=L_{1} \sqcup \cdots \sqcup L_{t}$ such that for each $m=1, \ldots, t$, the paths $\left(K \eta_{i}\right)\left(p_{l}\right)$ for $l \in L_{m}$ are all equal, and $\sum_{l \in L_{m}} \alpha_{l}=0$. There is an index $j \geq i$ in $I$ such that for each $m=1, \ldots, t$, the paths $\left(K \phi_{i j}\right)\left(p_{l}\right)$ for $l \in L_{m}$ are all equal. Hence, $\left(K \phi_{i j}\right)(b)=\sum_{l \in L} \alpha_{l}\left(K \phi_{i j}\right)\left(p_{l}\right)=0$ and so $a=\theta_{j}\left(K \phi_{i j}\right)(b)=0$. Thus $\psi$ is an isomorphism, as announced.

Therefore, we have shown that $K[-]$ preserves direct limits.

Now assume that $\mathcal{E}$ is a direct system in $\mathbf{C K G r}$, that is, all the maps $\phi_{i j}$ are CKmorphisms. As noted above, the limit maps $\eta_{i}$ are now CK-morphisms. Taking doubles (which is functorial), we obtain a direct system $\widehat{\mathcal{E}}=\left(\left(\widehat{E}_{i}\right)_{i \in I},\left(\widehat{\phi}_{i j}\right)_{i \leq j \text { in } I}\right)$, which lives in $\mathbf{D i G r}_{0}$. By what we have proved so far, $K \widehat{E}_{\infty}$ is a direct limit for $K[\widehat{\mathcal{E}}]$, with limit maps $K \widehat{\eta}_{i}: K \widehat{E}_{i} \rightarrow K \widehat{E}_{\infty}$.

For $i \leq j$ in $I$, we have $\pi_{K, E_{j}}\left(K \widehat{\phi}_{i j}\right)=L_{K}\left(\phi_{i j}\right) \pi_{K, E_{i}}$ and so $\left(K \widehat{\phi}_{i j}\right)\left(\operatorname{ker} \pi_{K, E_{i}}\right) \subseteq$ $\operatorname{ker} \pi_{K, E_{j}}$. Similarly, $\left(K \widehat{\eta}_{i}\right)\left(\operatorname{ker} \pi_{K, E_{i}}\right) \subseteq \operatorname{ker} \pi_{K, E_{\infty}}$ for all $i \in I$. We claim that any of the standard generators of $\operatorname{ker} \pi_{K, E_{\infty}}$ must lie in $\left(K \widehat{\eta}_{i}\right)\left(\operatorname{ker} \pi_{K, E_{i}}\right)$ for some $i$. First, for any $e \in E_{\infty}^{1}$, we have $e=\eta_{i}^{1}\left(e_{i}\right)$ for some $i \in I$ and some $e_{i} \in E_{i}^{1}$, whence $\left(K \widehat{\eta}_{i}\right)\left(e_{i} e_{i}^{*}-r\left(e_{i}\right)\right)=$ $e e^{*}-r(e)$. Similarly, any distinct edges $e, f \in E_{\infty}^{1}$ arise as $e=\eta_{i}^{1}\left(e_{i}\right)$ and $f=\eta_{i}^{1}\left(f_{i}\right)$ for 
some $i \in I$ and some distinct edges $e_{i}, f_{i} \in E_{i}^{1}$, whence $\left(K \widehat{\eta}_{i}\right)\left(e_{i} f_{i}^{*}\right)=e f^{*}$. Finally, consider a vertex $v \in E_{\infty}^{0}$ which is neither a sink nor an infinite emitter. As in the proof of part (a), there exist $i \in I$ and a vertex $w \in E_{i}^{0}$ such that $\eta_{i}^{0}(w)=v$ and $\eta_{i}^{1}$ sends the edges emitted by $w$ bijectively onto the edges emitted by $v$. Thus,

$$
v-\sum_{e \in E_{\infty}^{1}, s(e)=v} e e^{*}=\left(K \widehat{\eta}_{i}\right)\left(w-\sum_{f \in E_{i}^{1}, s(f)=w} f f^{*}\right),
$$

and the claim is proved. It follows that

$$
\operatorname{ker} \pi_{K, E_{\infty}}=\bigcup_{i \in I}\left(K \widehat{\eta}_{i}\right)\left(\operatorname{ker} \pi_{K, E_{i}}\right)
$$

Therefore $K \widehat{E}_{\infty} / \operatorname{ker} \pi_{K, E_{\infty}}$ is the direct limit of the quotients $K \widehat{E}_{i} / \operatorname{ker} \pi_{K, E_{i}}$, that is, $L_{K}\left(E_{\infty}\right)$ is the direct limit of the system $L_{K}(\mathcal{E})$ in $\mathbf{C K G r}$.

Proposition 2.6. [Ara-Moreno-Pardo] If $E$ is a row-finite graph, then $E$ is the direct limit (in $\mathbf{C K G r}$ ) of its finite CK-subgraphs. Consequently, the Leavitt path algebra of E, over any field $K$, is the direct limit of the Leavitt path algebras of the finite CK-subgraphs of $E$.

Proof. As noted in (2.3), the CK-morphisms between row-finite graphs coincide with the complete graph homomorphisms in the sense of [10]. In particular, the CK-subgraphs of $E$ are just the complete subgraphs of $[\mathbf{1 0}]$. Hence, the proposition is just a restatement of $[\mathbf{1 0}$, Lemmas 3.1, 3.2].

In general, Proposition 2.6 fails for row-infinite graphs, since such a graph may have too meager a supply of finite CK-subgraphs. For instance, take the graph $E$ consisting of one vertex $v$ and infinitely many edges (all of which must be loops from $v$ to $v$ ); the only finite CK-subgraphs of $E$ are the empty graph (with no vertices or edges) and the graph with one vertex $v$ but no edges. However, there is a general result for direct limits of countable subgraphs, as follows.

Proposition 2.7. Let $E$ be an arbitrary graph, and $K$ a field. Then $E$ is the direct limit of its countable CK-subgraphs, and consequently $L_{K}(E)$ is the direct limit of the $L_{K}(F)$ over countable CK-subgraphs $F$ of $E$.

Proof. For the first conclusion, we just need to show that $E$ is the directed union of its countable CK-subgraphs, i.e., each vertex or edge of $E$ lies in at least one countable CKsubgraph, and any two countable CK-subgraphs of $E$ are contained in a common countable CK-subgraph. Once this is established, the second conclusion follows via Lemma 2.5(b). The required properties are both consequences of the following claim:

- Claim. Given any countable subsets $X^{0} \subseteq E^{0}$ and $X^{1} \subseteq E^{1}$, there exists a countable CK-subgraph $F$ of $E$ such that $X^{0} \subseteq F^{0}$ and $X^{1} \subseteq F^{1}$. 
Starting with $X^{0}$ and $X^{1}$ as in the claim, we construct a countable ascending sequence of countable subgraphs of $E$, labelled $F_{0} \subseteq F_{1} \subseteq \cdots$. To begin, let $F_{0}$ be the subgraph of $E$ generated by $X^{0} \sqcup X^{1}$, that is, $F_{0}^{1}=X^{1}$ and $F_{0}^{0}=X^{0} \sqcup r_{E}\left(X^{1}\right) \cup s_{E}\left(X^{1}\right)$, with $r_{F_{0}}$ and $s_{F_{0}}$ being the restrictions of $r_{E}$ and $s_{E}$ to $F_{0}^{1}$.

Once $F_{n}$ has been constructed, choose sets $X_{n}^{1}(v) \subseteq E^{1}$ for each vertex $v \in F_{n}^{0}$ as follows:

- If $v$ emits at most finitely many edges in $E$, set $X_{n}^{1}(v)=s_{E}^{-1}(v)$;

- If $v$ emits infinitely many edges in $E$, take $X_{n}^{1}(v)$ to be some countably infinite subset of $s_{E}^{-1}(v)$.

Then, set $F_{n+1}$ equal to the subgraph of $E$ generated by $F_{n}^{0} \sqcup F_{n}^{1} \cup \bigcup_{v \in F_{n}^{0}} X_{n}^{1}(v)$.

Finally, $F=\bigcup_{n=0}^{\infty} F_{n}$ is a countable CK-subgraph of $E$ containing $X^{0} \sqcup X^{1}$.

2.8. Some Notation. Since we will utilize the direct limits given in Proposition 2.7 a number of times, it is convenient to establish some corresponding notation. Given a graph $E$, write $\left(E_{\alpha}\right)_{\alpha \in A}$ for the family of all countable CK-subgraphs of $E$. Inclusions among these subgraphs translate into a partial ordering on the index set $A$, where $\alpha, \beta \in A$ satisfy $\alpha \leq \beta$ if and only if $E_{\alpha} \subseteq E_{\beta}$. As shown in the proof of Proposition 2.7, any countable subgraph of $E$ is contained in some $E_{\alpha}$. In particular, this means that $A$ is countably upward directed: given any countable sequence $\alpha_{1}, \alpha_{2}, \ldots$ in $A$, there is some $\alpha \in A$ such that all $\alpha_{i} \leq \alpha$. Let us also observe that any nonempty countable upward directed subset $B \subseteq A$ has a supremum in $A$. To see this, we just need to observe that the union of the subgraphs indexed by $B$ is a countable CK-subgraph of $E$. In particular, any ascending sequence $\alpha_{1} \leq \alpha_{2} \leq \cdots$ in $A$ has a supremum.

For $\alpha \in A$, let $\eta_{\alpha}$ denote the inclusion map $E_{\alpha} \rightarrow E$, and for $\alpha \leq \beta$ in $A$, let $\phi_{\alpha \beta}$ denote the inclusion map $E_{\alpha} \rightarrow E_{\beta}$. Then we have

$$
E=\lim _{\longrightarrow}\left(\left(E_{\alpha}\right)_{\alpha \in A},\left(\phi_{\alpha \beta}\right)_{\alpha \leq \beta \text { in } A}\right),
$$

with limit maps $\eta_{\alpha}$. For any field $K$, we then have

$$
L_{K}(E)=\lim _{\longrightarrow}\left(\left(L_{K}\left(E_{\alpha}\right)\right)_{\alpha \in A},\left(L_{K}\left(\phi_{\alpha \beta}\right)\right)_{\alpha \leq \beta \text { in } A}\right),
$$

with limit maps $L_{K}\left(\eta_{\alpha}\right)$.

\section{IDEALS}

A number of key results about the ideal theory of Leavitt path algebras of countable graphs are established in [24] and [3]. While some of the proofs work equally well for uncountable graphs, the main results rely on a process called desingularization, introduced in [16], which only applies to graphs in which each vertex emits at most countably many edges. A desingularization of a countable graph $E$ is a countable row-finite graph $E^{\prime}$, such that for any field $K$, the algebras $L_{K}(E)$ and $L_{K}\left(E^{\prime}\right)$ are Morita equivalent [3, Theorem 5.2] (see [24, Lemma 6.7] for another proof). Since the proof of this theorem relies on the countability of $E^{0}$, there is at present no usable theory of desingularization for uncountable graphs. The general theorems, however, can be extended to uncountable graphs by direct limit techniques, as we show below. We begin with a rough outline of a key point of the procedure. 
3.1. Modus Operandi. Let $E$ be a graph, expressed as a direct limit of its countable CK-subgraphs as in (2.8). For suitable "finitely definable" graph-theoretic properties $\mathcal{P}$ (a term we do not make completely precise), a sufficient supply of countable CK-subgraphs of $E$ satisfying $\mathcal{P}$ can be constructed as follows.

Assume that $\mathcal{P}$ can be expressed in the form "for any choice of finitely many vertices and edges satisfying a certain finite list of conditions, there exist finitely many vertices and edges satisfying another given finite list of conditions". If $E$ satisfies $\mathcal{P}$, then given any finite subset $X$ of $E$ (that is, $X \subseteq E^{0} \sqcup E^{1}$ ) satisfying the hypotheses of $\mathcal{P}$, there is a finite subgraph $F$ of $E$ that contains $X$ together with a finite subset satisfying the conclusions of $\mathcal{P}$. Given any index $\alpha \in A$, the countable subgraph $E_{\alpha}$ has only countably many finite subsets, and so $E$ has a countable subgraph $G$ that contains $E_{\alpha}$ along with finite subsets satisfying the conclusions of $\mathcal{P}$ relative to any finite subset of $E_{\alpha}$. Since every countable subgraph of $E$ is contained in a countable CK-subgraph, $G \subseteq E_{\beta}$ for some $\beta \geq \alpha$ in $A$. Now repeat this procedure countably many times, obtaining indices $\beta(0)=\alpha \leq \beta(1) \leq \ldots$ in $A$ such that for each finite subset $X$ of any $E_{\beta(n)}$ satisfying the hypotheses of $\mathcal{P}$, there is a finite subset of $E_{\beta(n+1)}$ satisfying the conclusions of $\mathcal{P}$ relative to $X$. Finally, taking $\beta$ to be the supremum of the $\beta(n)$ in $A$, we observe that $E_{\beta}=\bigcup_{n=0}^{\infty} E_{\beta(n)}$ satisfies $\mathcal{P}$.

Therefore, if the property $\mathcal{P}$ behaves as required in the above outline, we conclude that for each $\alpha \in A$, there is some $\beta \geq \alpha$ in $A$ such that $E_{\beta}$ satisfies $\mathcal{P}$. Consequently, $E$ is a direct limit in $\mathbf{C K G r}$ of countable graphs satisfying $\mathcal{P}$.

As a first illustration of our M.O., we consider the homogeneous ideals of Leavitt path algebras $L_{K}(E)$, with respect to the $\mathbb{Z}$-grading introduced in (1.4). (In many references, these ideals are called graded rather than homogeneous.) Tomforde proved in [24, Theorem 4.8] that if $E$ is countable, $\pi$ is a graded ring homomorphism from $L_{K}(E)$ to a $\mathbb{Z}$-graded ring, and $\pi(v) \neq 0$ for all $v \in E^{0}$, then $\pi$ is injective. An equivalent conclusion is that every nonzero homogeneous ideal of $L_{K}(E)$ has nonempty intersection with $E^{0}$. Tomforde's proof, which does not use desingularization, appears to work for uncountable graphs just as well as for countable ones. That involves checking through a long sequence of details, however. As direct limits offer a quick means to carry over the result from the countable to the uncountable case, we prove it that way, to show off the method.

Theorem 3.2. (Extending [24, Theorem 4.8]) Let $K$ be a field and $E$ a graph. Then every nonzero homogeneous ideal of $L_{K}(E)$ has nonempty intersection with $E^{0}$.

Proof. Write $E=\underline{\lim } E_{\alpha}$ and $L_{K}(E)=\lim _{\longrightarrow} L_{K}\left(E_{\alpha}\right)$ as in (2.8), and let $I$ be a nonzero homogeneous ideal of $L_{K}(E)$. Each of the limit maps $L_{K}\left(\eta_{\alpha}\right)$ is a graded homomorphism, whence $I_{\alpha}=L_{K}\left(\eta_{\alpha}\right)^{-1}(I)$ is a homogeneous ideal of $L_{K}\left(E_{\alpha}\right)$. Further, $I=$ $\bigcup_{\alpha \in A} L_{K}\left(\eta_{\alpha}\right)\left(I_{\alpha}\right)$, and so there must be some $\beta \in A$ such that $I_{\beta} \neq 0$. By [24, Theorem 4.8], there exists a vertex $v \in I_{\beta} \cap E_{\beta}^{0}$, whence $L_{K}\left(\eta_{\beta}\right)(v) \in I \cap E^{0}$.

Corollary 3.3. Let $K$ be a field. For any morphism $\phi: E \rightarrow F$ in $\mathbf{C K G r}$, the $K$-algebra homomorphism $L_{K}(\phi): L_{K}(E) \rightarrow L_{K}(F)$ is injective.

Proof. Since $L_{K}(\phi)$ is a graded ring homomorphism, its kernel is a homogeneous ideal of $L_{K}(E)$. Moreover, $L_{K}(\phi)$ maps any vertex $v \in E^{0}$ to a vertex $\phi^{0}(v) \in F^{0}$, and so 
$L_{K}(\phi)(v) \neq 0$ by Lemma 1.5. Therefore Theorem 3.2 implies ker $L_{K}(\phi)=0$.

In order to address arbitrary (non-homogeneous) ideals of Leavitt path algebras, some graph-theoretic properties must be considered.

3.4. Conditions $(\mathbf{K})$ and $(\mathbf{L})$. Let $E$ be a graph. A path $p=e_{1} e_{2} \cdots e_{n}$ in $E$ is closed if $r(p)=s(p)$, in which case $p$ is said to be based at the vertex $r(p)=s(p)$. A closed path $p$ as above is simple provided it does not pass through its base more than once, i.e., $s\left(e_{i}\right) \neq s\left(e_{1}\right)=s(p)$ for all $i=2, \ldots, n$. More strictly, $p$ is a cycle if it is closed and it does not pass through any vertex twice, i.e., $s\left(e_{i}\right) \neq s\left(e_{j}\right)$ for all $i \neq j$. An exit for $p$ is an edge $e \in E^{1}$ that starts at some vertex on $p$ but does not coincide with the next edge of $p$, i.e., there is an index $i \in\{1, \ldots, n\}$ such that $s(e)=s\left(e_{i}\right)$ but $e \neq e_{i}$. (However, it is allowed that $r(e)$ might equal some $s\left(e_{j}\right)$.) Note that if $p$ is a simple closed path which is not a cycle, then $p$ automatically has an exit: There are indices $1<i<j \leq n$ such that $s\left(e_{i}\right)=s\left(e_{j}\right)$ but $e_{i} \neq e_{j}$, so $e_{j}$ is an exit at $s\left(e_{i}\right)$.

The graph $E$ satisfies Condition $(\mathrm{K})$ provided no vertex $v \in E^{0}$ is the base of precisely one simple closed path, i.e., either no simple closed paths are based at $v$, or at least two are based there. It satisfies Condition (L) provided every simple closed path in $E$ has an exit, or, equivalently, every cycle in $E$ has an exit.

Observe that $(\mathrm{K}) \Longrightarrow(\mathrm{L})$. For if $p=e_{1} e_{2} \cdots e_{n}$ is a cycle in $E$, then Condition $(\mathrm{K})$ implies that $E$ contains a simple closed path $q=f_{1} f_{2} \cdots f_{m} \neq p$ based at $s(p)$. We cannot have $e_{i}=f_{i}$ for $i \leq \min \{m, n\}$, because then the longer of $p$ or $q$ would pass through $s(p)=s(q)$ more than once. Take $j \leq \min \{m, n\}$ to be the least index such that $e_{j} \neq f_{j}$. Then $s\left(e_{j}\right)=s\left(f_{j}\right)$ and $f_{j}$ is an exit for $p$.

Lemma 3.5. Let $E$ be a graph that satisfies Condition (K) (respectively, Condition (L)). Write $E=\lim E_{\alpha}$ as in (2.8). For each $\alpha \in A$, there exists $\beta \geq \alpha$ in $A$ such that $E_{\beta}$ satisfies Condition $(\mathrm{K})$ (respectively, Condition (L)). Consequently, $E$ is a direct limit in CKGr of countable graphs satisfying Condition (K) (respectively, Condition (L)).

Proof. Assume first that $E$ satisfies Condition $(\mathrm{K})$. Given $\alpha \in A$, let $v_{1}, v_{2}, \ldots$ be a list of those vertices in $E_{\alpha}^{0}$ which are bases for simple closed paths in $E_{\alpha}$. For $i=1,2, \ldots$, there is a simple closed path $p_{i}$ in $E_{\alpha}$ based at $v_{i}$, and since $E$ satisfies Condition $(\mathrm{K})$, there must be a simple closed path $q_{i} \neq p_{i}$ in $E$ which is based at $v_{i}$. There is some $\gamma \geq \alpha$ in $A$ such that $E_{\gamma}$ contains all the $q_{i}$, and thus no vertex in $E_{\alpha}^{0}$ is the base of precisely one simple closed path in $E_{\gamma}$. Continuing this process as outlined in (3.1), we find that there is some $\beta \geq \alpha$ in $A$ such that $E_{\beta}$ satisfies Condition $(\mathrm{K})$.

Now suppose that $E$ satisfies Condition (L). Given $\alpha \in A$, let $p_{1}, p_{2}, \ldots$ be a list of all the cycles in $E_{\alpha}$. (There are at most countably many.) Each $p_{i}$ has an exit in $E$, say $e_{i}$. There is some $\gamma \geq \alpha$ in $A$ such that $E_{\gamma}$ contains all the $e_{i}$, and thus each cycle in $E_{\alpha}$ has an exit in $E_{\gamma}$. As before, it follows that there is some $\beta \geq \alpha$ in $A$ such that $E_{\beta}$ satisfies Condition (L).

We now extend Tomforde's Cuntz-Krieger Uniqueness Theorem to uncountable graphs.

Theorem 3.6. (Extending [24, Theorem 6.8, Corollary 6.10]) Let $K$ be a field and $E$ a graph satisfying Condition (L). Then every nonzero ideal of $L_{K}(E)$ has nonempty intersec- 
tion with $E^{0}$. Consequently, any ring homomorphism $\theta: L_{K}(E) \rightarrow R$ satisfying $\theta(v) \neq 0$ for all $v \in E^{0}$ must be injective.

Proof. Write $E=\lim _{\longrightarrow} E_{\alpha}$ as in (2.8), and set $\Gamma=\left\{\gamma \in A \mid E_{\gamma}\right.$ satisfies Condition (L) $\}$. In view of Lemma 3.5, $E$ is the directed union of the subgraphs $E_{\gamma}$ for $\gamma \in \Gamma$, and so $L_{K}(E)$ is a direct limit of the algebras $L_{K}\left(E_{\gamma}\right)$. By [24, Corollary 6.10], when $\gamma \in \Gamma$, every nonzero ideal of $L_{K}\left(E_{\gamma}\right)$ has nonempty intersection with $E_{\gamma}^{0}$. The theorem follows, just as in the proof of Theorem 3.2.

In order to adapt our modus operandi to ring-theoretic properties, we need to express Leavitt path algebras as directed unions of countable subrings which are themselves Leavitt path algebras. To do so, we just combine unions of countable subgraphs with unions of countable subfields.

3.7. Reduction to Countable Subfields. Let Fld denote the category of fields and Rng the category of arbitrary rings. In Rng, arbitrary maps preserving addition and multiplication are allowed as morphisms, while in Fld, morphisms must be unital (in order to qualify as field homomorphisms). Thus, Fld is a subcategory of Rng, but not a full one. Both of these categories have arbitrary direct limits. There is an obvious functor

\section{$L:$ Fld $\times$ CKGr $\longrightarrow$ Rng}

such that $L(K, E)=L_{K}(E)$ for any field $K$ and any graph $E$. If $(\phi, \psi):(K, E) \rightarrow(M, F)$ is a morphism in Fld $\times \mathbf{C K G r}$, then $L(\phi, \psi): L_{K}(E) \rightarrow L_{M}(F)$ is the composition of $L_{K}(\phi)$ with the map $L_{K}(F) \rightarrow M \otimes_{K} L_{K}(F) \equiv L_{M}(F)$ given by extension of scalars. We observe that the functor $L$ preserves direct limits.

When these tools are to be used, we modify the notation of (2.8) in the following way. Given a field $K$ and a graph $E$, write $\left(\left(K_{\alpha}, E_{\alpha}\right)\right)_{\alpha \in A}$ for the family of ordered pairs combining a countable subfield of $K$ with a countable CK-subgraph of $E$. For $\alpha, \beta \in A$, define $\alpha \leq \beta$ if and only if $K_{\alpha} \subseteq K_{\beta}$ and $E_{\alpha} \subseteq E_{\beta}$. As before, any countable ascending sequence in $A$ has a supremum.

For $\alpha \in A$, let $\lambda_{\alpha}$ and $\eta_{\alpha}$ denote the respective inclusion maps $K_{\alpha} \rightarrow K$ and $E_{\alpha} \rightarrow E$, and abbreviate $L\left(\lambda_{\alpha}, \eta_{\alpha}\right)$ to $L\left(\eta_{\alpha}\right)$. Similarly, for $\alpha \leq \beta$ in $A$, let $\kappa_{\alpha \beta}$ and $\phi_{\alpha \beta}$ denote the respective inclusion maps $K_{\alpha} \rightarrow K_{\beta}$ and $E_{\alpha} \rightarrow E_{\beta}$, and abbreviate $L\left(\kappa_{\alpha \beta}, \phi_{\alpha \beta}\right)$ to $L\left(\phi_{\alpha \beta}\right)$. Then we have

$$
(K, E)=\lim _{\longrightarrow}\left(\left(\left(K_{\alpha}, E_{\alpha}\right)\right)_{\alpha \in A},\left(\left(\kappa_{\alpha \beta}, \phi_{\alpha \beta}\right)\right)_{\alpha \leq \beta \text { in } A}\right)
$$

in Fld $\times \mathbf{C K G r}$, with limit maps $\left(\lambda_{\alpha}, \eta_{\alpha}\right)$, and

$$
L_{K}(E)=\lim _{\longrightarrow}\left(\left(L_{K_{\alpha}}\left(E_{\alpha}\right)\right)_{\alpha \in A},\left(L\left(\phi_{\alpha \beta}\right)\right)_{\alpha \leq \beta \text { in } A}\right)
$$

in $\mathbf{R n g}$, with limit maps $L\left(\eta_{\alpha}\right)$.

The M.O. of (3.1) readily adapts to ring-theoretic properties in the setting of (3.7), as in the following proof. 
Theorem 3.8. (Extending [24, Theorem 6.16]) Let $K$ be a field and $E$ a graph. Then all ideals of $L_{K}(E)$ are homogeneous if and only if $E$ satisfies Condition $(\mathrm{K})$.

Proof. $(\Longleftarrow)$ : Write $E=\lim E_{\alpha}$ as in (2.8), and let $\Gamma$ denote the set of those $\gamma \in A$ such that $E_{\gamma}$ satisfies Condition $(\mathrm{K})$. In view of Lemma 3.5, $E$ is the directed union of the subgraphs $E_{\gamma}$ for $\gamma \in \Gamma$, and so $L_{K}(E)$ is a direct limit of the algebras $L_{K}\left(E_{\gamma}\right)$, with limit maps $L_{K}\left(\eta_{\gamma}\right)$.

If $I$ is an ideal of $L_{K}(E)$, each $I_{\gamma}=L_{K}\left(\eta_{\gamma}\right)^{-1}(I)$ is an ideal of $L_{K}\left(E_{\gamma}\right)$, and $I=$ $\bigcup_{\gamma \in \Gamma} L_{K}\left(\eta_{\gamma}\right)\left(I_{\gamma}\right)$. By [24, Theorem 6.16], each $I_{\gamma}$ is a homogeneous ideal of $L_{K}\left(E_{\gamma}\right)$, and so $L_{K}\left(\eta_{\gamma}\right)\left(I_{\gamma}\right)$ is a homogeneous $K$-subspace of $L_{K}(E)$. Thus their union, $I$, is a homogeneous ideal.

$(\Longrightarrow)$ : This time, write $(K, E)=\lim \left(K_{\alpha}, E_{\alpha}\right)$ and $L_{K}(E)=\lim L_{K_{\alpha}}\left(E_{\alpha}\right)$ as in (3.7). Let us use the notations $\langle-\rangle_{\alpha}$ and $\langle-\rangle_{\infty}$ for ideals in $L_{K_{\alpha}}\left(E_{\alpha}\right)$ and $L_{K}(E)$, respectively.

Given $\alpha \in A$, a finite subset $X$ of $L_{K_{\alpha}}\left(E_{\alpha}\right)$, and an element $y \in\langle X\rangle_{\alpha}$, we have $L\left(\eta_{\alpha}\right)(y) \in\left\langle L\left(\eta_{\alpha}\right)(X)\right\rangle_{\infty}$, and so the homogeneous components of $L\left(\eta_{\alpha}\right)(y)$ all lie in $\left\langle L\left(\eta_{\alpha}\right)(X)\right\rangle_{\infty}$. At most finitely many of these components are nonzero, and so there exists $\gamma \geq \alpha$ in $A$ such that the homogeneous components of $L\left(\phi_{\alpha \gamma}\right)(y)$ all lie in $\left\langle L\left(\phi_{\alpha \gamma}\right)(X)\right\rangle_{\gamma}$. Now $L_{K_{\alpha}}\left(E_{\alpha}\right)$ has only countably many finite subsets, and each ideal of $L_{K_{\alpha}}\left(E_{\alpha}\right)$ has only countably many elements, so in view of the previous observations, there exists $\delta \geq \alpha$ in $A$ such that for all finite subsets $X \subseteq L_{K_{\alpha}}\left(E_{\alpha}\right)$ and all elements $y \in\langle X\rangle_{\alpha}$, the homogeneous components of $L\left(\phi_{\alpha \delta}\right)(y)$ all lie in $\left\langle L\left(\phi_{\alpha \delta}\right)(X)\right\rangle_{\delta}$. Repeating this procedure countably many times and taking the supremum of the resulting indices, we obtain an index $\beta \geq \alpha$ in $A$ such that all finitely generated ideals of $L_{K_{\beta}}\left(E_{\beta}\right)$ are homogeneous. Consequently, all ideals of $L_{K_{\beta}}\left(E_{\beta}\right)$ are homogeneous.

Combining the above result with [24, Theorem 6.16], we conclude that for each $\alpha \in$ $A$, there exists $\beta \geq \alpha$ in $A$ such that $E_{\beta}$ satisfies Condition $(\mathrm{K})$. Therefore $E$ satisfies Condition $(\mathrm{K})$.

3.9. Saturated Hereditary Sets of Vertices. Let $E$ be a graph and $H$ a subset (possibly empty) of $E^{0}$. We say that $H$ is hereditary if all edges leaving $H$ end in $H$, that is, whenever $e \in E^{1}$ and $s(e) \in H$, then also $r(e) \in H$. The set $H$ is called saturated provided that

- Each vertex $v \in E^{0}$ which is neither a sink nor an infinite emitter, and which satisfies $r(e) \in H$ for all $e \in s_{E}^{-1}(v)$, must lie in $H$.

Observe that any intersection of saturated hereditary subsets of $E^{0}$ is again hereditary and saturated. Hence, for any subset $V \subseteq E^{0}$, we can define the saturated hereditary subset of $E^{0}$ generated by $V$ as the smallest saturated hereditary subset containing $V$. This set can be described as follows.

Observation 3.10. Let $E$ be a graph, $V \subseteq E^{0}$, and $w \in E^{0}$. Then $w$ lies in the saturated hereditary subset of $E^{0}$ generated by $V$ if and only if there exists a finite sequence $w_{0}, w_{1}, \ldots, w_{n}=w$ in $E^{0}$ such that $w_{0} \in V$ and for all $i=1, \ldots, n$, one of the following holds:

(1) $w_{i} \in V$; 
(2) There exists an edge in $E^{1}$ from $w_{i-1}$ to $w_{i}$;

(3) $w_{i}$ is neither a sink nor an infinite emitter, and $r(e) \in\left\{w_{0}, \ldots, w_{i-1}\right\}$ for all $e \in s_{E}^{-1}\left(w_{i}\right)$.

Abrams and Aranda Pino characterized simplicity of Leavitt path algebras of countable row-finite graphs in [1, Theorem 3.11]. They later removed the row-finiteness hypothesis [3, Theorem 3.1], as did Tomforde, independently [24, Theorem 6.18].

Theorem 3.11. (Extending [3, Theorem 3.1; 24, Theorem 6.18]) Let $K$ be a field and $E$ a graph. Then $L_{K}(E)$ is a simple ring if and only if E satisfies Condition (L) and the only saturated hereditary subsets of $E^{0}$ are $\varnothing$ and $E^{0}$.

Proof. $(\Longleftarrow)$ : Write $E=\underset{\lim }{\longrightarrow} E_{\alpha}$ and $L_{K}(E)=\underset{\lim }{\longrightarrow} L_{K}\left(E_{\alpha}\right)$ as in (2.8). Lemma 3.5 shows that for each $\alpha \in A$, there exists $\beta \geq \alpha$ in $A$ such that $E_{\beta}$ satisfies Condition (L).

If $\alpha \in A$ and $v \in E_{\alpha}^{0}$, the saturated hereditary subset of $E^{0}$ generated by $v$ must equal $E^{0}$. In view of Observation 3.10, we see that for each $w \in E_{\alpha}^{0}$, there exists $\gamma \geq \alpha$ in $A$ such that $w$ lies in the saturated hereditary subset of $E_{\gamma}^{0}$ generated by $v$. Following the M.O. of (3.1), we conclude that for each $\alpha \in A$, there exists $\beta \geq \alpha$ in $A$ such that the saturated hereditary subset of $E_{\beta}^{0}$ generated by any vertex equals $E_{\beta}^{0}$.

Now merge the above results as follows. Given $\alpha \in A$, there is an ascending sequence $\alpha \leq \beta(1) \leq \beta(2) \leq \cdots$ in $A$ such that the graphs $E_{\beta(i)}$ for $i$ even satisfy condition (L), while those for $i$ odd satisfy the condition of the previous paragraph. The supremum of the $\beta(i)$ is then an index $\beta \geq \alpha$ in $A$ such that $E_{\beta}$ satisfies Condition (L) and the saturated hereditary subset of $E_{\beta}^{0}$ generated by any vertex equals $E_{\beta}^{0}$. In particular, the only saturated hereditary subsets of $E_{\beta}^{0}$ are $\varnothing$ and $E_{\beta}^{0}$. Combining this result with [3, Theorem $3.1 ; \mathbf{2 4}$, Theorem 6.18], we see that for each $\alpha \in A$, there exists $\beta \geq \alpha$ in $A$ such that $L_{K}\left(E_{\beta}\right)$ is a simple ring. Therefore $L_{K}(E)$ must be simple.

$(\Longrightarrow)$ : Now write $(K, E)=\underset{\lim }{\longrightarrow}\left(K_{\alpha}, E_{\alpha}\right)$ and $L_{K}(E)=\underset{\longrightarrow}{\lim } L_{K_{\alpha}}\left(E_{\alpha}\right)$ as in (3.7). As in the proof of the previous theorem, we will use the notations $\langle-\rangle_{\alpha}$ and $\langle-\rangle_{\infty}$ for ideals in $L_{K_{\alpha}}\left(E_{\alpha}\right)$ and $L_{K}(E)$, respectively.

We claim that for each $\alpha \in A$, there exists $\beta \geq \alpha$ in $A$ such that $L_{K_{\beta}}\left(E_{\beta}\right)$ is a simple ring. By our usual M.O., it is enough to show that for any $\alpha \in A$ and any nonzero $x, y \in$ $L_{K_{\alpha}}\left(E_{\alpha}\right)$, there is some $\gamma \geq \alpha$ in $A$ such that $L\left(\phi_{\alpha \gamma}\right)(x) \in\left\langle L\left(\phi_{\alpha \gamma}\right)(y)\right\rangle_{\gamma}$. But since $L\left(\eta_{\alpha}\right)$ is injective (Corollary 3.3), the element $L\left(\eta_{\alpha}\right)(y) \in L_{K}(E)$ is nonzero, whence $L\left(\eta_{\alpha}\right)(x) \in$ $\left\langle L\left(\eta_{\alpha}\right)(y)\right\rangle_{\infty}$. Since $L_{K}(E)=\underset{\lim }{\longrightarrow} L_{K_{\alpha}}\left(E_{\alpha}\right)$, it follows that $L\left(\phi_{\alpha \gamma}\right)(x) \in\left\langle L\left(\phi_{\alpha \gamma}\right)(y)\right\rangle_{\gamma}$ for some $\gamma \geq \alpha$ in $A$, as desired.

Combining the above claim with $[\mathbf{3}$, Theorem $3.1 ; \mathbf{2 4}$, Theorem 6.18$]$, we find that for each $\alpha \in A$, there is some $\beta \geq \alpha$ in $A$ such that $E_{\beta}$ satisfies Condition (L). It immediately follows that $E$ satisfies Condition (L).

Suppose that $E^{0}$ contains a proper nonempty saturated hereditary subset $H$. Then there exist $\alpha \in A$ and $v, w \in E_{\alpha}^{0}$ such that $\eta_{\alpha}^{0}(v) \in H$ while $\eta_{\alpha}^{0}(w) \notin H$. In view of the claims above, we may assume that $L_{K_{\alpha}}\left(E_{\alpha}\right)$ is simple. Since $\eta_{\alpha}$ is a CK-morphism, the set $H_{\alpha}=\left(\eta_{\alpha}^{0}\right)^{-1}(H)$ is a saturated hereditary subset of $E_{\alpha}^{0}$. But $v \in H_{\alpha}$ while $w \notin H_{\alpha}$, 
which contradicts $\left[\mathbf{3}\right.$, Theorem $3.1 ; \mathbf{2 4}$, Theorem 6.18]. Therefore $E^{0}$ contains no proper nonempty saturated hereditary subsets.

\section{ExChANGE Rings}

4.1. Exchange Rings. In the unital setting, exchange rings are rings over which the regular representation (the standard free module of rank 1) satisfies the exchange property in direct sum decompositions. The definition of the exchange property and many consequences can be found in numerous papers, of which we mention [25], [22], and the survey [7]. For present purposes, the key point is that exchange rings can be described by finitely many ring-theoretic equations [19, p. 167; 22, Theorem 2.1]: A unital ring $R$ is an exchange ring if and only if for each $x \in R$, there exists an idempotent $e \in x R$ such that $1-e \in(1-x) R$. Non-unital exchange rings were introduced by Ara [6], who defined a ring $R$ to be an exchange ring provided that for each $x \in R$, there exist elements $e, r, s \in R$ such that $e$ is an idempotent and $e=x r=x+s-x s$. In case $R$ is a ring which is generated as an ideal by its idempotents, it follows from [8, Theorem 3.3] that $R$ is an exchange ring if and only if all the corners $e R e$, for idempotents $e \in R$, are (unital) exchange rings.

In [13, Theorem 4.5], Aranda Pino, Pardo, and Siles Molina showed that the Leavitt path algebra of a countable row-finite graph $E$, over any base field, is an exchange ring if and only if $E$ satisfies Condition $(\mathrm{K})$. The row-finiteness hypothesis was removed by Abrams and Aranda Pino in [3, Theorem 5.4]. We can now remove the countability assumption.

Theorem 4.2. (Extending [3, Theorem 5.4]) Let $K$ be a field and $E$ a graph. Then $L_{K}(E)$ is an exchange ring if and only if $E$ satisfies Condition $(\mathrm{K})$.

Proof. $(\Longleftarrow)$ : Write $E=\lim E_{\alpha}$ as in (2.8), and let $\Gamma$ denote the set of those $\gamma \in A$ such that $E_{\gamma}$ satisfies Condition $(\mathrm{K})$. In view of Lemma 3.5, $E$ is the directed union of the subgraphs $E_{\gamma}$ for $\gamma \in \Gamma$, and so $L_{K}(E)$ is a direct limit of the algebras $L_{K}\left(E_{\gamma}\right)$. Each $L_{K}\left(E_{\gamma}\right)$ is an exchange ring by [3, Theorem 5.4], and therefore $L_{K}(E)$ must be an exchange ring.

$(\Longrightarrow)$ : Now write $(K, E)=\underset{\longrightarrow}{\lim }\left(K_{\alpha}, E_{\alpha}\right)$ and $L_{K}(E)=\underset{\lim }{\longrightarrow} L_{K_{\alpha}}\left(E_{\alpha}\right)$ as in (3.7).

Given $\alpha \in A$ and $x \in L_{K_{\alpha}}\left(E_{\alpha}\right)$, there exist elements $e, r, s \in L_{K}(E)$ such that $e$ is an idempotent and $e=L\left(\eta_{\alpha}\right)(x) r=L\left(\eta_{\alpha}\right)(x)+s-L\left(\eta_{\alpha}\right)(x) s$. There exist $\gamma \geq \alpha$ in $A$ and $e^{\prime}, r^{\prime}, s^{\prime} \in L_{K_{\gamma}}\left(E_{\gamma}\right)$ such that $e^{\prime}$ is an idempotent and $e^{\prime}=L\left(\phi_{\alpha \gamma}\right)(x) r^{\prime}=$ $L\left(\phi_{\alpha \gamma}\right)(x)+s^{\prime}-L\left(\phi_{\alpha \gamma}\right)(x) s^{\prime}$. By our usual M.O., it follows that for each $\alpha \in A$, there is some $\beta \geq \alpha$ in $A$ such that $L_{K_{\beta}}\left(E_{\beta}\right)$ is an exchange ring. For each such $\beta$, the graph $E_{\beta}$ satisfies Condition (K) by [3, Theorem 5.4]. Therefore $E$ satisfies condition (K).

\section{Non-Stable K-Theory}

The group $K_{0}$ of a ring $R$, precisely because it is a group, ignores direct sum cancellation questions. In particular, taking the unital case for the moment, finitely generated projective $R$-modules $A$ and $B$ represent the same class in $K_{0}(R)$ if and only if they are stably isomorphic, meaning that $A \oplus C \cong B \oplus C$ for some finitely generated projective $R$-module 
$C$. In order to keep track of isomorphism classes, one builds a monoid, denoted $V(R)$, in place of the group $K_{0}(R)$. This construction, which we now sketch, is a central object in what has become known as "non-stable K-theory".

5.1. $V(-)$. Let $I$ be a ring (with or without unit). There are two equivalent constructions of the monoid $V(I)$, one via projective modules over a unital extension ring of $I$, one via idempotent matrices. We begin with the second construction.

Write $a \oplus b$ for the block sum of square matrices $a$ and $b$, that is, for the matrix $\left(\begin{array}{ll}a & 0 \\ 0 & b\end{array}\right)$ where the 0 s are rectangular zero matrices of appropriate sizes. We can view each matrix ring $M_{n}(I)$ as a subring of $M_{n+1}(I)$ by identifying any $a \in M_{n}(I)$ with $a \oplus 0$. Set $M_{\infty}(I)=\bigcup_{n=1}^{\infty} M_{n}(I)$. In the "idempotent picture" of $V(I)$, the elements of $V(I)$ are equivalence classes $[e]$ of idempotents from $M_{\infty}(I)$, where idempotents $e$ and $f$ are equivalent if and only if there exist $a, b \in M_{\infty}(I)$ such that $a b=e$ and $b a=f$. This set of equivalence classes becomes an abelian monoid with the addition operation induced from block sums, that is, $[e]+[f]=[e \oplus f]$.

Functoriality of $V$ is clear from the above construction: Any morphism $\phi: I \rightarrow J$ in $\mathbf{R n g}$ induces a morphism $M_{\infty}(\phi): M_{\infty}(I) \rightarrow M_{\infty}(J)$ which preserves block sums, idempotents, and equivalence, so $M_{\infty}(\phi)$ in turn induces a monoid homomorphism $V(\phi): V(I) \rightarrow V(J)$. Thus, we obtain a functor $V(-)$ from Rng to the category of abelian monoids. It is a routine observation that this functor preserves direct limits.

For the "projective picture", choose any unital ring $R$ that contains $I$ as a two-sided ideal. Let $R$-Mod denote the usual category of unital left $R$-modules and module homomorphisms, and $\mathbf{F P}(I, R)$ the full subcategory of $R$-Mod whose objects are those finitely generated projective left $R$-modules $P$ such that $P=I P$. Then $V(I)$ can be defined as the monoid of isomorphism classes of objects in $\mathbf{F P}(I, R)$, with addition induced from direct sum. (In short, $V(I)$ is the Grothendieck monoid of the category $\mathbf{F P}(I, R)$.) There is a natural isomorphism from the previous incarnation of $V(I)$ to this one, under which the equivalence class of an idempotent $e \in M_{n}(I)$ is mapped to the isomorphism class of the module $R^{n} e$. In particular, this shows that, up to isomorphism, the projective module form of $V(I)$ does not depend on the choice of unital ring $R$ in which $I$ is embedded as an ideal.

The projective picture of $V(I)$ is convenient for dealing with Morita equivalence, as follows.

5.2. Nonunital Morita Equivalence. As in the unital case, Morita equivalence is based on equivalences of module categories. However, the category of arbitrary left modules over a non-unital ring $I$ is too large for the purpose - for one thing, it contains all abelian groups (viewed as $I$-modules with zero module multiplication). We follow the common practice (see [18], for instance) in defining $I$-Mod to be the category of those left $I$-modules $M$ which are

- full: $I M=M$, and

- nondegenerate: $I x=0$ implies $x=0$, for $x \in M$.

(The morphisms in $I$-Mod are arbitrary module homomorphisms between the above modules.) Observe that $I$-Mod has finite products (built as direct products) and arbitrary 
coproducts (built as direct sums).

Rings $I$ and $J$ are defined to be Morita equivalent provided the categories $I$-Mod and $J$-Mod are equivalent.

When $R$ and $S$ are Morita equivalent unital rings, the monoids $V(R)$ and $V(S)$ are clearly isomorphic - this follows easily from the projective picture, since the Morita equivalence implies that the categories of finitely generated projective modules over $R$ and $S$ are equivalent. In order to make a similar argument for nonunital rings, we need to deal with rings $I$ for which we can show that $V(I)$ is isomorphic to the monoid of isomorphism classes of objects from some categorically defined subcategory of $I$-Mod. Idempotent rings are suitable for this purpose, as follows.

5.3. Idempotent Rings. A ring $I$, when viewed as a left module over itself, might not be either full or nondegenerate, i.e., it can fail to be an object in I-Mod. Fullness occurs exactly when $I$ is idempotent, that is, $I^{2}=I$. Nondegeneracy can either be assumed, or obtained by factoring out a suitable ideal. If $I$ is idempotent and $J=\{x \in I \mid I x=0\}$, then $I / J$ is full and nondegenerate as either a left $I$-module or a left $(I / J)$-module.

Idempotence by itself is already helpful in working with $I$-Mod, as the following observation shows. We thank P. Ara for communicating it to us.

- If $I$ is idempotent, then every epimorphism in $I$-Mod is surjective.

Given an epimorphism $f: M \rightarrow N$ in $I$-Mod, set $X=\{x \in N \mid I x \subseteq f(M)\}$ and observe that $N / X$ is nondegenerate. It is also full, because $N$ is full, whence $N / X$ is an object in $I$-Mod. Now since $f$ is an epimorphism, the quotient map $N \rightarrow N / X$ must coincide with the zero map, and thus $N / X=0$. Consequently, $N=I N \subseteq f(M)$, proving that $f$ is surjective.

The dual statement also holds:

- If $I$ is idempotent, then every monomorphism in $I$-Mod is injective.

Given a monomorphism $g: M \rightarrow N$ in $I$-Mod, set $K=g^{-1}(\{0\})$ (the usual kernel). Then $I K$ is a full, nondegenerate submodule of $M$, and hence an object in $I$-Mod. Now since $g$ is a monomorphism, the inclusion map $I K \rightarrow M$ must coincide with the zero map, whence $I K=0$. Nondegeneracy then implies $K=0$, proving that $g$ is injective.

As above, assume that $I$ is idempotent, and set $J=\{x \in I \mid I x=0\}$. Note that $J^{2}=0$. If $M$ is a nondegenerate left $I$-module, then $I J M=0$ implies $J M=0$, so that $M$ is, in a canonical way, a left $(I / J)$-module. Consequently, the objects in $I$-Mod can be identified with the objects in $(I / J)$-Mod, and thus we can identify these two categories. Finally, we observe that factoring out $J$ does not harm $V(I)$ :

- $V(I) \cong V(I / J)$.

More precisely, $V(\pi): V(I) \rightarrow V(I / J)$ is an isomorphism, where $\pi$ denotes the quotient map $I \rightarrow I / J$. To see that $V(\pi)$ is surjective, it suffices to lift idempotents from any matrix ring $M_{n}(I / J)$ to $M_{n}(I)$. Since $M_{n}(J)^{2}=0$, this is a classical fact (e.g., see [20, p. 72 , Proposition 1] and note that the proof works just as well in the nonunital case). For injectivity, we need to show that if $e$ and $f$ are any idempotents in some $M_{n}(I)$ whose images are equivalent in $M_{n}(I / J)$, then $e$ and $f$ are equivalent. This is also a classical fact, 
but we have not located a convenient reference, so we sketch a proof. By assumption, there exist $a, b \in M_{n}(I)$ such that $a b-e$ and $b a-f$ lie in $M_{n}(J)$. After replacing $a$ and $b$ by $e a f$ and $f b e$, we may assume that $a=e a f$ and $b=f b e$. Now the element $a b \in e M_{n}(I) e$ is the sum of $e$ plus a nilpotent, so it is a unit in that ring. Set $c=b(a b)^{-1}$, so that $c=f c e$ and $a c=e$. Since $b a$ is the sum of $f$ plus a nilpotent, it is a unit in $f M_{n}(I) f$. Consequently, we can cancel the left hand $a$ factor from $a c a=e a=a f$ to conclude that $c a=f$. Therefore $e$ and $f$ are equivalent, as needed.

5.4. Compact Objects. Recall that an object $C$ in a category $\mathbf{M}$ with coproducts and a zero object is said to be compact provided the following property holds: Given any set $\left(X_{\alpha}\right)_{\alpha \in A}$ of objects in $\mathbf{M}$ and any epimorphism $f: \coprod_{\alpha \in A} X_{\alpha} \rightarrow C$, there exists a finite subset $B \subseteq A$ such that the composition of $f$ with the natural map $\coprod_{\beta \in B} X_{\beta} \rightarrow \coprod_{\alpha \in A} X_{\alpha}$ is an epimorphism.

Lemma 5.5. Let $I$ be an idempotent ring. Then $V(I)$ is isomorphic to the monoid of isomorphism classes of compact projective objects of I-Mod (with addition induced from direct sum).

Proof. Set $J=\{x \in I \mid I x=0\}$. As noted in (5.3), $J$ is an ideal of $I$ such that $I / J$ is nondegenerate as a left module over itself, $V(I) \cong V(I / J)$, and $I$-Mod $=(I / J)$-Mod. Thus, after replacing $I$ by $I / J$, we may assume that $I$ is a nondegenerate left $I$-module.

Let $R$ be the canonical unitification of $I$, namely the unital ring containing $I$ as a twosided ideal such that $R=\mathbb{Z} \oplus I$. The forgetful functor provides a category isomorphism from $R$-Mod to the category of arbitrary left $I$-modules [17, Proposition 8.29B], and we identify these two categories. Then $I$-Mod is identified with the full subcategory of $R$-Mod whose objects are the full nondegenerate left $I$-modules.

We claim that the objects of $\mathbf{F P}(I, R)$ are precisely the compact projective objects of $I$-Mod. Once this is proved, the lemma follows.

First, let $P$ be an object in $\mathbf{F P}(I, R)$. By definition, $P$ is a full $I$-module. Further, $P$ is isomorphic to an $R$-submodule of a direct sum of copies of $R$, so $P=I P$ is also isomorphic to an $I$-submodule of a direct sum of copies of $I$. Since $I$ is a nondegenerate module over itself, $P$ is a nondegenerate $I$-module. Hence, $P$ is an object of $I$-Mod. Projectivity of $P$ in $R$-Mod, together with the surjectivity of epimorphisms in $I$-Mod, now implies that $P$ is projective in $I$-Mod.

Suppose that $\left(X_{\alpha}\right)_{\alpha \in A}$ is a set of objects in $I$-Mod and $f: \coprod_{\alpha \in A} X_{\alpha} \rightarrow P$ an epimorphism. We may view this coproduct as an internal direct sum of modules. Since $f$ is surjective, $\sum_{\alpha \in A} f\left(X_{\alpha}\right)=P$. Each $f\left(X_{\alpha}\right)$ is an $R$-submodule of $P$, and so ( $P$ being finitely generated) there must be a finite subset $B \subseteq A$ such that $\sum_{\beta \in B} f\left(X_{\beta}\right)=P$. The composition of $f$ with the natural map $\coprod_{\beta \in B} X_{\beta} \rightarrow \coprod_{\alpha \in A} X_{\alpha}$ is thus an epimorphism, proving that $P$ is a compact object of $I$-Mod.

Conversely, let $P$ be an arbitrary compact projective object of $I$-Mod. Since $\sum_{x \in P} I x=$ $I P=P$, it follows from the compactness of $P$ and the surjectivity of epimorphisms in $I$-Mod that there are finitely many elements $x_{1}, \ldots, x_{n} \in P$ such that $\sum_{j=1}^{n} I x_{j}=P$. In particular, $\sum_{j=1}^{n} R x_{j}=P$, and so $P$ is finitely generated as an $R$-module. Choose a free $R$-module $F$ and an epimorphism $f: F \rightarrow P$ in $R$-Mod. Then $I F$ is a full nondegenerate 
$I$-module, and $f$ restricts to a surjective $I$-module homomorphism $f^{\prime}: I F \rightarrow P$. Since $P$ is projective in $I$-Mod, there is an $I$-module homomorphism $h: P \rightarrow I F$ such that $f^{\prime} h=\operatorname{id}_{P}$. But $h$ can also be viewed as an $R$-module homomorphism $P \rightarrow F$ satisfying $f h=\operatorname{id}_{P}$, proving that $P$ is a projective $R$-module. Thus, $P$ is an object of $\mathbf{F P}(I, R)$, and the claim is established.

The following corollary of Lemma 5.5 is well known among certain researchers, but has not appeared in the literature to our knowledge.

Corollary 5.6. If $I$ and $J$ are Morita equivalent idempotent rings, then $V(I) \cong V(J)$.

Proof. Since $I$-Mod and $J$-Mod are equivalent, so are their full subcategories of compact projective objects.

5.7. Refinement Monoids. Let $V$ be an abelian monoid, written additively. It is called a refinement monoid provided it satisfies the Riesz refinement property: Whenever $x_{1}, x_{2}, y_{1}, y_{2} \in V$ with $x_{1}+x_{2}=y_{1}+y_{2}$, there exist elements $z_{i j} \in V$ for $i, j=1,2$ such that $z_{i 1}+z_{i 2}=x_{i}$ for $i=1,2$ while $z_{1 j}+z_{2 j}=y_{j}$ for $j=1,2$. To describe some additional properties such a monoid might enjoy, it is convenient to equip $V$ with the algebraic preorder $\leq$ defined by the existence of subtraction, i.e., elements $x, y \in V$ satisfy $x \leq y$ if and only if there is some $v \in V$ such that $x+v=y$. This relation is reflexive, transitive, and invariant under translation, the latter meaning that $x \leq y$ implies $x+z \leq y+z$ for any $z \in V$.

The monoid $V$ is separative if it satisfies the following weak cancellation condition: $x+x=x+y=y+y$ implies $x=y$, for any $x, y \in V$. Equivalently, $V$ is separative if and only if $x+z=y+z$ implies $x=y$ for any $x, y, z \in V$ such that $z \leq n x$ and $z \leq n y$ for some $n \in \mathbb{N}[\mathbf{9}$, Lemma 2.1]. Finally, $V$ is said to be unperforated provided that $n x \leq n y$ implies $x \leq y$, for any $x, y \in V$ and any $n \in \mathbb{N}$.

Observe that refinement, separativity, and unperforation are all preserved in direct limits of monoids.

Ara, Moreno, and Pardo proved in [10] that for any row-finite graph $E$ and any field $K$, the monoid $V\left(L_{K}(E)\right)$ is an unperforated, separative refinement monoid. To remove the row-finiteness restriction, we use desingularizations to deal with countable graphs, followed by direct limits.

Theorem 5.8. (Extending [10, Corollary 6.5]) If $K$ is a field and $E$ a graph, then $V\left(L_{K}(E)\right)$ is an unperforated, separative refinement monoid.

Proof. Assume first that $E$ is countable. Then there exists a desingularization $E^{\prime}$ of $E$, which is a countable row-finite graph such that $L_{K}\left(E^{\prime}\right)$ is Morita equivalent to $L_{K}(E)[\mathbf{3}$, Theorem 5.2]. The desired properties hold for $L_{K}\left(E^{\prime}\right)$ by [10, Corollary 6.5], and they then transfer to $L_{K}(E)$ by Corollary 5.6.

In the general case, we have $L_{K}(E)=\underset{\lim }{\longrightarrow} L_{K}\left(E_{\alpha}\right)$ as in (2.8), where the $E_{\alpha}$ run over the countable CK-subgraphs of $E$. By the previous paragraph, refinement, separativity, and unperforation hold in each $V\left(L_{K}\left(E_{\alpha}\right)\right)$, and therefore they hold in $\lim V\left(L_{K}\left(E_{\alpha}\right)\right)$, which is isomorphic to $V\left(L_{K}(E)\right)$. 


\section{ACKNOWLEDGEMENTS}

The author thanks Gene Abrams, Pere Ara, and Gonzalo Aranda Pino for useful discussions, suggestions, and correspondence.

\section{REFERENCES}

1. G. Abrams and G. Aranda Pino, The Leavitt path algebra of a graph, J. Algebra 293 (2005), 319-334.

2. _ Purely infinite simple Leavitt path algebras, J. Pure Appl. Algebra 207 (2006), 553-563.

3. The Leavitt path algebras of arbitrary graphs, Houston J. Math. (to appear).

4. G. Abrams, G. Aranda Pino, F. Perera, and M. Siles Molina, Chain conditions for Leavitt path algebras, Preprint, 2007.

5. G. Abrams, G. Aranda Pino, and M. Siles Molina, Finite-dimensional Leavitt path algebras, J. Pure Appl. Algebra 209 (2007), 753-762.

6. P. Ara, Extensions of exchange rings, J. Algebra 197 (1997), 409-423.

7. _ Stability properties of exchange rings, in Internat. Symposium on Ring Theory (Kyongju 1999), Birkhäuser, Boston, 2001, pp. 23-42.

8. P. Ara, M. Gómez Lozano, and M. Siles Molina, Local rings of exchange rings, Communic. in Algebra 26 (1998), 4191-4205.

9. P. Ara, K. R. Goodearl, K. C. O'Meara, and E. Pardo, Separative cancellation for projective modules over exchange rings, Israel J. Math. 105 (1998), 105-137.

10. P. Ara, M. A. Moreno, and E. Pardo, Nonstable K-Theory for graph algebras, Algebras Represent. Theory 10 (2007), 157-178.

11. P. Ara and E. Pardo, Stable rank of Leavitt path algebras, Proc. Amer. Math. Soc. (to appear).

12. G. Aranda Pino, D. Martín Barquero, C. Martín González, and M. Siles Molina, The socle of a Leavitt path algebra (to appear).

13. G. Aranda Pino, E. Pardo and M. Siles Molina, Exchange Leavitt path algebras and stable rank, J. Algebra 305 (2006), 912-936.

14. G. Aranda Pino, F. Perera and M. Siles Molina, Graph Algebras: Bridging the Gap between Analysis and Algebra, University of Málaga Press, Málaga, Spain, 2007.

15. J. Cuntz, Simple $C^{*}$-algebras generated by isometries, Comm. Math. Phys. 57 (1977), $173-185$.

16. D. Drinen and $M$. Tomforde, The $C^{*}$-algebras of arbitrary graphs, Rocky Mountain J. Math. 35 (2005), 105-135.

17. C. Faith, Algebra: Rings, Modules and Categories I, Springer-Verlag, New York, 1973.

18. J. L. García and J. J. Simón, Morita equivalence for idempotent rings, J. Pure Appl. Algebra 76 (1991), 39-56.

19. K. R. Goodearl and R. B. Warfield, Jr., Algebras over zero-dimensional rings, Math. Ann. 223 (1976), 157-168.

20. J. Lambek, Lectures on Rings and Modules, Blaisdell, Waltham, 1966.

21. W. G. Leavitt, The module type of a ring, Trans. Amer. Math. Soc. 42 (1962), 113-130.

22. W. K. Nicholson, Lifting idempotents and exchange rings, Trans. Amer. Math. Soc. 229 (1977), 269-278.

23. M. Siles Molina, Algebras of quotients of Leavitt path algebras (to appear).

24. M. Tomforde, Uniqueness theorems and ideal structure for Leavitt path algebras, J. Algebra 318 (2007), 270-299.

25. R. B. Warfield, Jr., Exchange rings and decompositions of modules, Math. Ann. 199 (1972), 31-36.

Department of Mathematics, University of California, Santa Barbara, CA 93106

E-mail address: goodearl@math.ucsb.edu 